\title{
Nickel-Catalyzed Synthesis of Primary Aryl and Heteroaryl Amines via C-O Bond Cleavage
}

\author{
Huifeng Yue, Lin Guo, Xiangqian Liu, and Magnus Rueping* \\ Institute of Organic Chemistry, RWTH Aachen University, Landoltweg 1, 52074 Aachen, Germany \\ KEYWORDS Amination $\bullet$ Aryl esters $\bullet$ C-O Bond Activation $\bullet$ benzophenone imine $\bullet$ Nickel Catalysis
}

\begin{abstract}
A nickel-catalyzed protocol for the conversion of aryl and heteroaryl alcohol derivatives to primary and secondary aromatic amines via $\mathrm{C}\left(\mathrm{sp}^{2}\right)$-O bond cleavage is described. The new amination protocol can be applied to a range of substrates bearing diverse functional groups and uses readily available benzophenone imines as an effective nitrogen source.
\end{abstract}

Aromatic amines are highly valuable intermediates which play pivotal roles in the preparation of pharmaceutical agents, agrochemicals, dyes and polymers. ${ }^{1}$ The most common ways to access this class of compounds employ transition metalcatalyzed amination reactions of aryl derivatives, especially aryl halides. ${ }^{2,3}$ Although these methods proved to be convenient for the synthesis of amines, it is desirable to develop more efficient and environmentally benign protocols which make use of easily available catalysts and substrates to further extend the scope of these coupling reactions. In recent years, phenol derivatives have emerged as versatile, green and inexpensive alternatives to aryl halides in coupling chemistry due to their lack of toxicity and readily availability. ${ }^{4}$ To overcome the challenges associated with the high activation energy required for the $\mathrm{C}\left(\mathrm{sp}^{2}\right)-\mathrm{O}$ bond scission, nickel catalysis which became a powerful synthetic tools for cross-coupling reactions, is usually employed. ${ }^{5}$ Recently, amination protocols for the construction of $\mathrm{C}\left(\mathrm{sp}^{2}\right)-\mathrm{N}$ bonds have been described. ${ }^{6}$ These protocols are, however, limited to the formation of secondary and tertiary amines. The Ni-catalyzed synthesis of primary amines, one of the most important classes of nitrogencontaining compounds, which can undergo further modifications, has been addressed starting mainly from aryl halides. ${ }^{7}$

In this context, the development of a general protocol, making use of easily accessible phenol derivatives as electrophiles is highly desirable, in particular if one could directly access primary amines. Given the enormous interest in efficient protocols for the preparation of amines we decided to examine a new nickel catalyzed amination procedure. In order to achieve the synthesis of the anilines from anisoles we decided to apply imines as the nitrogen nucleophiles. This would allow a further functionalization as well as direct access to primary as well as secondary aromatic amines. Herein, we report a general protocol for the nickel-catalyzed synthesis of primary aromatic amines via inert $\mathrm{C}-\mathrm{O}$ bond cleavage (Scheme 1).

$$
\begin{aligned}
& \mathrm{Ar}-\mathrm{OR}+\mathrm{HN}=\overbrace{\mathrm{R}^{1}}^{\mathrm{R}^{2}} \frac{\text { Nickel catalysis }}{\text { hydrolysis }} \mathrm{Ar}-\mathrm{NH}_{2} \\
& \mathrm{R}=\mathrm{CO}^{t} \mathrm{Bu}, \mathrm{CO}_{2}{ }^{t} \mathrm{Bu}, \mathrm{CONEt}_{2}, \mathrm{SO}_{2} \mathrm{NMe}_{2}
\end{aligned}
$$

Scheme 1. Primary and secondary amines formation.

We started our investigations by choosing 2-naphthyl pivalate (2a) as model substrate and benzophenone imine in the presence of nickel catalyst. We anticipated that 1,3-bis(2,6diisopropylphenyl)-imidazolium chloride (IPr $\cdot \mathrm{HCl})$ would be a suitable ligand for our desired amination cross-coupling reaction. However, the product was obtained only in traces when $\mathrm{IPr} \cdot \mathrm{HCl}$ was used with $\mathrm{Ni}(\operatorname{cod})_{2}$ as catalyst and $\mathrm{Cs}_{2} \mathrm{CO}_{3}$ as base (Table 1, entry 1 ). Also, the most commonly used phosphine ligands such as $\mathrm{PCy}_{3}$, dppe, dppf and dcypf were found inefficient, giving only traces or no product (entries 2-5). Nevertheless, the $\mathrm{Ni}(\operatorname{cod})_{2}$ /dcype system was able to catalyze the amination reaction in toluene in the presence of $\mathrm{Cs}_{2} \mathrm{CO}_{3}$ and provided 3a in a high yield of $89 \%$ (entry 6), which indicated that this amination protocol was strongly liganddependent. Encouraged by this result, we further optimized other reaction parameters. The use of other bases instead of $\mathrm{Cs}_{2} \mathrm{CO}_{3}$ led to lower yield $\left(\mathrm{CsF}, \mathrm{K}_{2} \mathrm{CO}_{3}\right.$, and $\left.\mathrm{K}_{3} \mathrm{PO}_{3}\right)$ or no yield $\left(\mathrm{Na}_{2} \mathrm{CO}_{3}\right)$, which indicated that the base is crucial for this reaction (entries 7-10). Presumably, the better solubility of $\mathrm{Cs}_{2} \mathrm{CO}_{3}$ is the reason for improved yield. Among the solvents examined (toluene, dioxane, THF), toluene gave the best result (entries 5, 11 and 12). Other nitrogen nucleophiles such as ammonia and diphenylmethanamine were found to be not suitable for this transformation under the optimized reaction conditions (entries 13 and 14). Control experiments showed that the coupling reaction did not occur in the absence of $\mathrm{Ni}(\operatorname{cod})_{2}$ (Table 1, entry 13). After surveying various ligands, bases and solvents, the optimal reaction conditions were assigned as follows: $\mathrm{Ni}(\mathrm{cod})_{2}$, dcype as ligand, $\mathrm{Cs}_{2} \mathrm{CO}_{3}$ as base in toluene at $100{ }^{\circ} \mathrm{C}$ for $12 \mathrm{~h}$. 
Table 1. Optimization of the reaction conditions. ${ }^{a}$

\begin{tabular}{|c|c|c|c|c|}
\hline $1 a$ & $\begin{array}{r}+\mathrm{HN}= \\
2 \mathrm{a}\end{array}$ & $\begin{array}{l}\text { 1. } \mathrm{Ni}(\mathrm{cod})_{2} \\
\text { ligand }(\mathrm{x} \\
\text { base }(2 \\
\text { solvent, }\end{array}$ & $\begin{array}{l}\text { mol\%) } \\
\%) \\
\text { v) } \\
{ }^{\circ} \mathrm{C}, 12 \mathrm{~h} \\
\underset{\text { lysis }}{\longrightarrow}\end{array}$ & $\mathbf{a}$ \\
\hline Entry & $\begin{array}{l}\text { Ligand } \\
(\mathrm{x} \mathrm{mol \% )}\end{array}$ & Base & Solvent & $\begin{array}{l}\text { Yield } \\
(\%)^{b}\end{array}$ \\
\hline 1 & $\mathrm{IPr} \cdot \mathrm{HCl}(20)$ & $\mathrm{Cs}_{2} \mathrm{CO}_{3}$ & toluene & Trace \\
\hline 2 & $\mathrm{PCy}_{3}(20)$ & $\mathrm{Cs}_{2} \mathrm{CO}_{3}$ & toluene & - \\
\hline 3 & dppe (10) & $\mathrm{Cs}_{2} \mathrm{CO}_{3}$ & toluene & - \\
\hline 4 & dppf (10) & $\mathrm{Cs}_{2} \mathrm{CO}_{3}$ & toluene & Trace \\
\hline 5 & dcypf (10) & $\mathrm{Cs}_{2} \mathrm{CO}_{3}$ & toluene & Trace \\
\hline 6 & dcype (10) & $\mathrm{Cs}_{2} \mathrm{CO}_{3}$ & toluene & 89 \\
\hline 7 & dcype (10) & $\mathrm{CsF}$ & toluene & 32 \\
\hline 8 & dcype (10) & $\mathrm{K}_{2} \mathrm{CO}_{3}$ & toluene & 17 \\
\hline 9 & dcype (10) & $\mathrm{K}_{3} \mathrm{PO}_{4}$ & toluene & 42 \\
\hline 10 & dcype (10) & $\mathrm{Na}_{2} \mathrm{CO}_{3}$ & toluene & - \\
\hline 11 & dcype (10) & $\mathrm{Cs}_{2} \mathrm{CO}_{3}$ & Dioxane & 63 \\
\hline 12 & dcype (10) & $\mathrm{Cs}_{2} \mathrm{CO}_{3}$ & $\mathrm{THF}$ & 45 \\
\hline $13^{c}$ & dcype (10) & $\mathrm{Cs}_{2} \mathrm{CO}_{3}$ & toluene & - \\
\hline $14^{d}$ & dcype (10) & $\mathrm{Cs}_{2} \mathrm{CO}_{3}$ & toluene & 10 \\
\hline $15^{e}$ & - & $\mathrm{Cs}_{2} \mathrm{CO}_{3}$ & toluene & - \\
\hline
\end{tabular}

${ }^{a}$ Reaction conditions: naphthyl pivalate 1a $(0.2 \mathrm{mmol})$, benzophenone imine $\mathbf{2 a}(0.3 \mathrm{mmol}, 1.5$ equiv. $), \mathrm{Ni}(\mathrm{cod})_{2}(0.02$ $\mathrm{mmol})$, ligand $(0.02 \mathrm{mmol}$ or $0.04 \mathrm{mmol})$, base $(0.4 \mathrm{mmol})$ in the indicated solvent $(1 \mathrm{ml})$ at $100{ }^{\circ} \mathrm{C}$. ${ }^{b}$ Yield of isolated products. ${ }^{c} \mathrm{NH}_{3}$ (0.3 mmol, $0.5 \mathrm{M}$ in 1,4-dioxane) was used instead of $\mathbf{2 a}$. ${ }^{d}$ Diphenylmethanamine $(0.3 \mathrm{mmol})$ was used instead of $\mathbf{2 a}$. ${ }^{e}$ Without $\mathrm{Ni}(\operatorname{cod})_{2} . \mathrm{Piv}=$ pivalate .<smiles></smiles>

$\mathrm{IPr} \cdot \mathrm{HCl}$

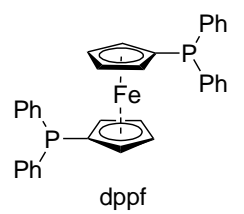<smiles>[PbH3]</smiles>

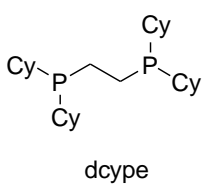
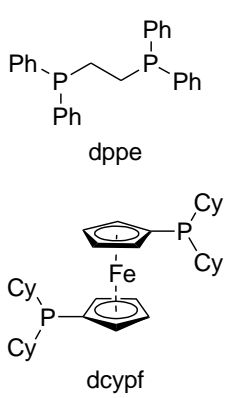

With the optimized reaction conditions in hand, the functional group tolerance of the amination reaction was firstly examined. As shown in Table 2, benzophenone imine 2a was coupled with a variety of substituted naphthyl pivalates in good yields. In addition, not only pivalates but also carbonate, carbamate and sulphamate derivatives of 2-naphthol could be converted into the desired primary amine 3a. However, 2methoxynaphthalene and 2-ethoxynaphthalene bearing more inert C-O bonds failed to give the desired product. Similarly, the use of naphthalen-1-yl pivalate also gave the corresponding product $\mathbf{3 b}$ in $80 \%$ yield. The chemoselectivity profile of this method was nicely illustrated by the fact that functional groups such as methoxy (3c, 3d, 3h), ester (3e), cyano (3f), fluoride (3i), pyrazole $(\mathbf{3 j})$ and amide $(\mathbf{3 k})$ were well tolerated under the present conditions.

Table 2. Scope of the naphthyl pivalates. ${ }^{a}$
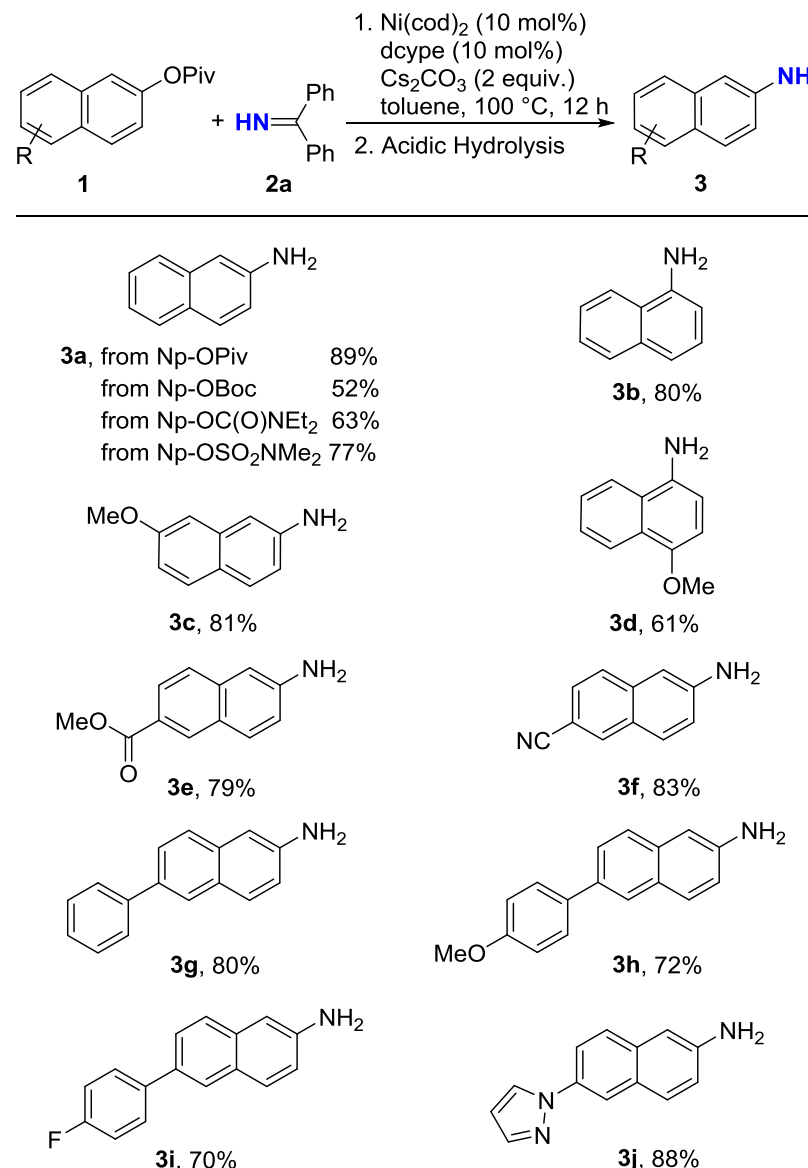

3b, $80 \%$<smiles>COc1ccc(N)c2ccccc12</smiles>

3d, $61 \%$<smiles>N#Cc1ccc2cc(N)ccc2c1</smiles>

3f, $83 \%$<smiles>COc1ccc(-c2ccc3cc(N)ccc3c2)cc1</smiles>

$3 h, 72 \%$<smiles>Nc1ccc2cc(-n3cccn3)ccc2c1</smiles>

$3 \mathbf{j}, 88 \%$<smiles>Nc1ccc2cccc(N=P)c2c1</smiles>

$3 \mathbf{k}, 66 \%$<smiles>Nc1ccc2cccc(N)c2c1</smiles>

$31,79 \%^{b}$<smiles>Nc1cc2ccccc2c2ccccc12</smiles>

$3 \mathrm{~m}, 75 \%$<smiles>Cc1cc(N)c2ccccc2n1</smiles>

3o, $92 \%$<smiles>Nc1cccc2cccnc12</smiles>

$3 n, 63 \%$

$3 p, 52 \%$<smiles>Nc1cnc2ccccc2n1</smiles>

${ }^{a}$ Reaction conditions: (hetero)aryl pivalate 1 (0.2 $\left.\mathrm{mmol}\right)$, benzophenone imine $\mathbf{2 a}(0.3 \mathrm{mmol}), \mathrm{Ni}(\mathrm{cod})_{2}(0.02 \mathrm{mmol})$, dcype $(0.02 \mathrm{mmol}), \mathrm{Cs}_{2} \mathrm{CO}_{3}(0.4 \mathrm{mmol})$ in toluene $(1 \mathrm{ml})$ at $100{ }^{\circ} \mathrm{C}$. ${ }^{b} \mathrm{Ni}(\mathrm{cod})_{2}(20 \mathrm{~mol} \%)$, dcype $(20 \mathrm{~mol} \%)$, benzophenone imine $2 \mathbf{a}$ (3 equiv.), $\mathrm{Cs}_{2} \mathrm{CO}_{3}$ (4 equiv.), from the corresponding bispivalate.

Moreover, the amination reaction could also be applied with good results to larger conjugated systems (1g-i). In the case of aryl bispivalate substrate $\mathbf{1 1}$, the amination took place twice to 
produce diamine $\mathbf{3 1}$ in $79 \%$ yield. A polycyclic substrate such as phenanthren-9-pivalate also gave the product $\mathbf{3 m}$ in $75 \%$ yield. It is noteworthy that heterocyclic substrates were also suitable for this catalytic transformation, affording the corresponding quinoline amines in moderate to excellent yields (3n-p). Regarding the use of simple phenol derivatives, this type of substrates proved to be more challenging for our amination protocol. Strikingly, our Ni-catalyzed amination reaction took place efficiently with 4-cyanophenyl pivalate, giving amine $\mathbf{5 a}$ in $78 \%$ yield. In addition, switching the substrate from phenyl pivalate to phenyl sulfamate and raising the reaction temperature to $120{ }^{\circ} \mathrm{C}$ allowed this amination process to occur and the products were obtained in moderate to high yields (5b-e).

Table 3. Scope of the phenyl sulfamates. ${ }^{a}$

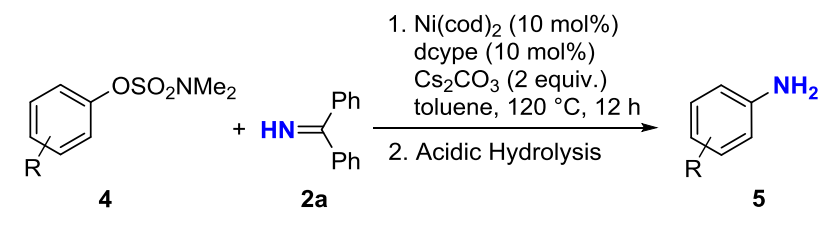

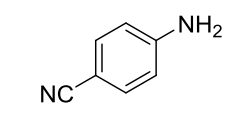

5 a, $78 \%^{b}$<smiles>Nc1ccccc1-c1ccccc1</smiles>

5b, $80 \%$

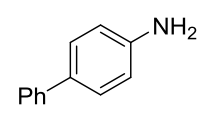

5c, $71 \%$<smiles>COC(=O)c1ccc(N)cc1</smiles>

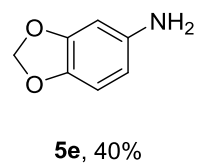

${ }^{a}$ Reaction conditions: aryl sulfamate $4 \quad(0.2 \quad \mathrm{mmol})$, benzophenone imine $2 \mathrm{a}(0.3 \mathrm{mmol}), \mathrm{Ni}(\mathrm{cod})_{2}(0.02 \mathrm{mmol})$, dcype $(0.02 \mathrm{mmol}), \mathrm{Cs}_{2} \mathrm{CO}_{3}(0.4 \mathrm{mmol})$ in toluene $(1 \mathrm{ml})$ at $120{ }^{\circ} \mathrm{C}$. ${ }^{b}$ From the corresponding pivalate, $100{ }^{\circ} \mathrm{C}$.

Table 4. Nickel catalyzed secondary amine formation with different N-H imines. ${ }^{a}$
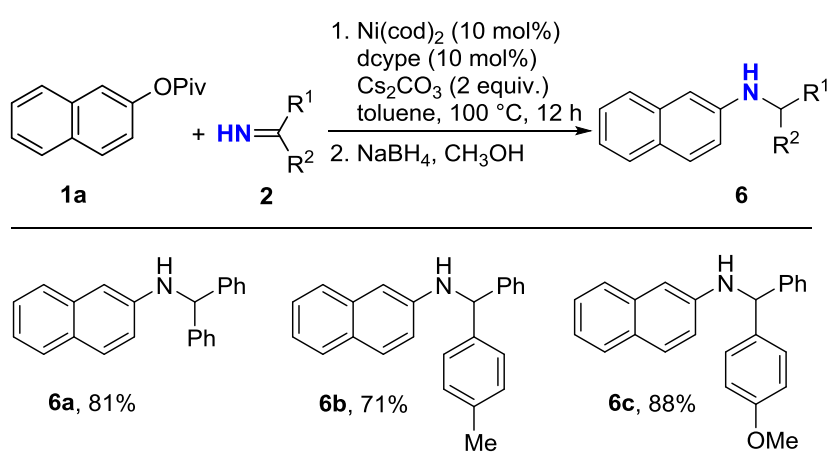

${ }^{a}$ Reaction conditions: naphthyl pivalate $1 \mathbf{a}(0.2 \mathrm{mmol})$, benzophenone imine $2(0.3 \mathrm{mmol}), \mathrm{Ni}(\mathrm{cod})_{2}(0.02 \mathrm{mmol})$, dcype $(0.02 \mathrm{mmol}), \mathrm{Cs}_{2} \mathrm{CO}_{3}(0.4 \mathrm{mmol})$ in toluene $(1 \mathrm{ml})$ at $100{ }^{\circ} \mathrm{C}$.

Subsequently, we examined the secondary amine formation procedure by reduction of the ketimine intermediates (Table 4). Gratifyingly, we observed that various imines could be cross-coupled with naphthyl pivalate (1a) under $\mathrm{Ni}(\mathrm{cod})_{2} /$ dcype catalytic system and then reduced by sodium borohydride to provide the corresponding secondary amines 6a-c in high yields.

In summary, we have developed a Ni-catalyzed cross-coupling reaction of aryl esters with benzophenone imines. The protocol we described herein constitutes the first general strategy for the preparation of primary aryl amines via Ni-catalyzed inert $\mathrm{C}-\mathrm{O}$ bond cleavage. Considering the substrate scope of this protocol, the method provides a new and efficient route to aryl amines from readily available aryl esters. The protocol shows good chemoselectivity and functional groups including fluoride, ether, or nitril groups previously used in crosscouplings remain intact. Since aryl and heteroaryl amines are highly valuable products for many different applications this new amination protocol will be of value and may become a good alternative to aryl halides amination reactions. Efforts to investigate the reaction mechanism as well as to broaden the applicability are currently ongoing in our laboratories.

\section{ASSOCIATED CONTENT}

Supporting Information. Detailed experimental procedures, spectral data for all compounds, and copies of ${ }^{1} \mathrm{H},{ }^{13} \mathrm{C}$, and ${ }^{19} \mathrm{~F}$ NMR spectra. This material is available free of charge via the Internet at http://pubs.acs.org.

\section{AUTHOR INFORMATION}

\section{Corresponding Author}

*magnus.rueping@rwth-aachen.de

\section{Author Contributions}

The manuscript was written through contributions of all authors. / All authors have given approval to the final version of the manuscript.

\section{Notes}

The authors declare no competing financial interests.

\section{ACKNOWLEDGMENT}

H. Yue, L. Guo and X. Liu were supported by the China Scholarship Council.

\section{REFERENCES}

(1) (a) Ricci, A., Ed.; Amino Group Chemistry: From Synthesis to the Life Sciences; Wiley-VCH: Weinheim, Germany, 2008. (b) Lawerence, S. A. Amines: Synthesis, Properties and Applications, Cambridge University, Cambridge, 2004. (c) Brown, B. R. The Organic Chemistry of Aliphatic Nitrogen Compounds, Cambridge University, Cambridge, 2004. (d) Rappoport, Z., Ed.; The Chemistry of Anilines, Parts 1 and 2; John Wiley \& Sons: New York, 2007. (e) Kienle, M.; Dubbaka, S. R.; Brade, K.; Knochel, P. Eur. J. Org. Chem. 2007, 4166-4176. (f) Liang, M.; Chen, J. Chem. Soc. Rev. 2013, 42, 3453-3488.

(2) (a) Wolfe, J. P.; Wagaw, S.; Marcoux, J. F.; Buchwald, S. L. Acc. Chem. Res. 1998, 31, 805-818. (b) Hartwig, J. F. Acc. Chem. Res. 1998, 31, 852-860. (c) Hartwig, J. F. Angew. Chem. Int. Ed. 1998, 37 2046-2067. (d) Muci, A. R.; Buchwald, S. L. Top. Curr. Chem. 2002, 219, 131-209. (e) Hartwig, J. F. Acc. Chem. Res. 2008, 41, 1534-1544. (f) Surry, D. S.; Buchwald, S. L. Angew. Chem. Int. Ed. 2008, 47, 6338-6361. (g) Surry, D. S.; Buchwald, S. L. Chem. Sci. 2011, 2, 2750. 
(3) (a) Wolfe, J. P.; Buchwald, S. L. J. Am. Chem. Soc. 1997, 119, 6054-6058. (b) Brenner, E.; Fort, Y. Tetrahedron Lett. 1998, 39, 5359-5362. (c) Brenner, E.; Schneider, R.; Fort, Y. Tetrahedron 1999, 55, 12829-12842. (d) Desmarets, C.; Schneider, R.; Fort, Y. J. Org. Chem. 2002, 67, 3029-3036. (e) Chen, C.; Yang, L.-M. J. Org. Chem. 2007, 72, 6324-6327. (f) Manolikakes, G.; Gavryushin, A.; Knochel, P. J. Org. Chem. 2008, 73, 1429-1434. (g) Gao, C.-Y.; Yang, L.-M. J. Org. Chem. 2008, 73, 1624-1627. (h) Gao, C.-Y.; Cao, X.; Yang, L.M. Org. Biomol. Chem. 2009, 7, 3922-3925. (i) Ackermann, L.; Song, W.; Sandmann, R. J. Organomet. Chem. 2011, 696, 195-201. (j) Martin, A. R.; Nelson, D. J.; Meiries, S.; Slawin, A. M. Z.; Nolan, S. P. Eur. J. Org. Chem. 2014, 3127-3131. (k) Kapmann, S. S.; Sobolev, A. N.; Koutsantonis, G. A.; Stewart, S. G. Adv. Synth. Catal. 2014, 356, 1967-1973. (1) Ge, S.; Green, R. A.; Hartwig, J. F. J. Am. Chem. Soc. 2014, 136, 1617-1627.

(4) (a) Yu, D. G.; Li, B. J.;. Shi, Z. J Acc. Chem. Res. 2010, 43, 1486-1495. (b) Li, B. J.; Yu, D. G.; Sun, C. L.; Shi, Z. J. Chem. Eur. J. 2011, 17, 1728-1759. (c) Rosen, B. M.; Quasdorf, K. W.; Wilson, D. A.; Zhang, N.; Resmerita, A. M.; Garg, N. K.; Percec, V. Chem. Rev. 2011, 111, 1346-1416. (d) Mesganaw, T.; Garg, N. K. Org. Process Res. Dev. 2013, 17, 29-39. (e) Cornella, J.; Zarate, C.; Martin, R. Chem. Soc. Rev. 2014, 43, 8081-8097. (f) Tobisu, M.; Chatani, N. Acc. Chem. Res. 2015, 48, 1717-1726. (g) Su, B.; Cao, Z.-C.; Shi, Z.J. Acc. Chem. Res. 2015, 48, 886-896. (h) S. I. Kozhushkov, H. K. Potukuchi, L. Ackermann, Catal. Sci. Technol. 2013, 3, 562-571.

(5) Examples: (a) Tamaru, Y. Modern Organonickel Chemistry, Wiley-VCH, Weinheim, Germany, 2005. (b) Phapale, V. B.; Cárdenas, D. J. Chem. Soc. Rev. 2009, 38, 1598-1607. (c) Yamaguchi, J.; Muto, K.; Itami, K. Eur. J. Org. Chem. 2013, 19-30. (d) Han, F.-S. Chem. Soc. Rev. 2013, 42, 5270-5298.

(6) (a) Tobisu, M.; Shimasaki, T.; Chatani, N. Chem. Lett. 2009, 38 , 710-711. (b) Tobisu, M.; Yasutome, A.; Yamakawa, K.; Shimasaki, T.; Chatani, N. Tetrahedron 2012, 68, 5157-5161. (c) Shimasaki, T.; Tobisu, M.; Chatani, N. Angew. Chem. Int. Ed. 2010, 49, 2929-2932. (d) Mesganaw, T.; Silberstein, A. L.; Ramgren, S. D.; Fine Nathel, N. F.; Hong, X.; Liu, P.; Garg, N. K. Chem. Sci. 2011, 2, 1766-1771. (e) Hie, L.; Ramgren, S. D.; Mesganaw, T.; Garg, N. K. Org. Lett. 2012, 14, 4182-4185. (f) Ramgren, S. D.; Silberstein, A. L.; Yang, Y.; Garg, N. K. Angew. Chem. Int. Ed. 2011, 50, 2171-2173. (g) Ackermann, L.; Sandmann, R.; Song, W. Org. Lett. 2011, 13, 1784-1786. (h) Fine Nathel, N. F.; Kim, J.; Hie, L.; Jiang, X.; Garg, N. K. ACS Catal. 2014, 4, 3289-3293. (i) Park, N. H.; Teverovskiy, G.; Buchwald, S. L. Org. Lett. 2014, 16, 220-223. (j) Hanley, P. S.; Clark, T. P.; Krasovskiy, A.; Ober, M. S.; O’Brien, J. P.; Staton, T. S. ACS Catal. 2016, 6, 3515-3519. (k) Huang, J.-H.; Yang, L.-M. Org. Lett. 2011, 13, 3750-3753. (1) Gao, C.-Y.; Yang, L.-M. J. Org. Chem. 2008, 73, 1624-1627. (m) Iglesias, M. J.; Blandez, J. F.; Fructos, M. R.; Prieto, A.; Álvarez, E.; Belderrain, T. R.; Nicasio, M. C. Organometallics 2012, 31, 6312-6316. (n) Kampmann, S. S.; Skelton, B. W.; Wild, D. A.; Koutsantonis, G. A.; Stewart, S. G. Eur. J. Org. Chem. 2015, 59956004. For a review, see: (o) Marin, M.; Rama, R. J.; Nicasio, M. C. Chem. Rec. 2016, 16, 1819-1832.

(7) (a) Green, R. A.; Hartwig, J. F. Angew. Chem. Int. Ed. 2015, 54, 3768-3772. (b) Borzenko, A.; Rotta-Loria, N. L.; MacQueen, P. M.; Lavoie, C. M.; McDonald, R.; Stradiotto, M. Angew. Chem. Int. Ed. 2015, 54, 3773-3777. (c) Lavoie, C. M.; MacQueen, P. M.; RottaLoria, N. L.; Sawatzky, R. S.; Borzenko, A.; Chisholm, A. J.; Hargreaves, B. K. V.; McDonald, R.; Ferguson, M. J.; Stradiotto, M. Nature Commun. 2016, 7, 11073. 
Insert Table of Contents artwork here

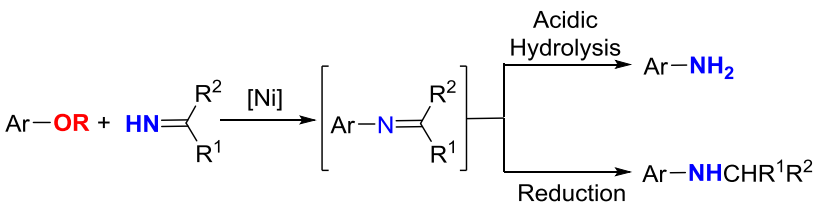

$$
\begin{aligned}
& \mathrm{R}=\mathrm{CO}^{t} \mathrm{Bu}, \mathrm{CO}_{2}{ }^{t} \mathrm{Bu}, \mathrm{CONEt}_{2}, \mathrm{SO}_{2} \mathrm{NMe}_{2} \\
& \text { Primary and Secondary } \\
& \text { Amines Formation }
\end{aligned}
$$

